\title{
5,6-DIHYDRO-[1,2,4]TRIAZOLO[1,5-c]QUINAZOLINES. MESSAGE 1. FEATURES OF INTERACTIONS BETWEEN [2-(3-ARYL-1 H-1,2,4-TRIAZOLE-5-YL)PHENYL]AMINES, ALIPHATIC AND AROMATIC ALDEHYDES
}

\author{
S.V.Kholodnyak, K.P.Schabelnyk, O.Yu.Voskoboynik, G.G.Berest, S.I.Kovalenko
}

Zaporizhzhia State Medical University

26, Mayakovsky av., Zaporizhzhia, 69035, Ukraine

Key words: 5-[2-(3-aryl-1H-1,2,4-triazol-5-yl)phenyl]amines; [5+1]cyclocondensation; spectral characteristics

\begin{abstract}
Reactions of [5+1]-cyclocondensation of [2-(3-aryl-1H-1,2,4-triazol-5-yl)phenyl]amines with aliphatic and aromatic aldehydes produce the corresponding 5-R-2-aryl-5,6-dihydro- or 5-R-2-aryl-[1,2,4]triazolo[1,5-c]quinazolines depending on the conditions. The optimal conditions of the reaction have been found, and factors contributing to oxidation of 5-R-2-aryl-5,6-dihydro-[1,2,4]triazolo[1,5-c]quinazolines have been determined. The alternative synthetic approaches for 5-R-2-aryl-[1,2,4]triazolo[1,5-c]quinazolines, namely oxidation of their reduced analogues and interaction of [2-(3-aryl-1H-1,2,4-triazol-5-yl)phenyl]amines with acylhalides of aliphatic or aromatic carboxylic acid have been proposed. Purity and the structure of the compounds synthesized have been confirmed by the complex of physicochemical methods, including LC-MS, ${ }^{1} \mathrm{H}-,{ }^{13} \mathrm{C} N M R$, mass-spectrometry and elemental analysis. The peculiarities and differences of ${ }^{1} \mathrm{H}$ - and ${ }^{13} \mathrm{C}-\mathrm{NMR}$ spectral patterns of $5-R-2-a r y l-5,6-$ dihydro-[1,2,4]triazolo[1,5-c]quinazolines and their aromatic analogues have been described.
\end{abstract}

5,6-ДИГІДРО-[1,2,4]ТРИАЗОЛО[1,5-С]ХІНАЗОЛІНИ. ПОВІДОМЛЕННЯ 1. ОСОБЛИВОСТІ ПОВЕДІНКИ [2-(3-АРИЛ1Н-1,2,4-ТРИАЗОЛ-5-ІЛ)ФЕНІЛ]АМІНІВ В РЕАКЦІЯХ З АЛЬДЕГІДАМИ АЛІФАТИЧНОГО ТА АРОМАТИЧНОГО РЯДУ С.В.Холодняк, К.П.Шабельник, О.Ю.Воскобойнік, Г.Г.Берест, С.І.Коваленко

Ключові слова: [2-(3-арил-1Н-1,2,4-триазол-5-іл)феніл]аміни; [5+1]-циклоконденсація; спектральні характеристики

Реакції [5+1]-циклоконденсації [2-(3-арил-1Н-1,2,4-триазол-5-іл)френіл]амінів з альдегідами аліфратичного та ароматичного ряду в залежності від умов проведення перебігають з утворенням 5-R-2-арил5,6-дигідро- або 5-R-2-арил-[1,2,4]триазоло[1,5-с]хіназолінів. Визначені оптимальні умови реакції та встановлені основні фрактори, які сприяють окисненню 5-R-2-арил-5, 6-дигідро-[1,2,4]триазоло[1,5-c] хіназолінів. Розроблені методи зустрічного синтезу 5-R-2-арил-[1,2,4]триазоло[1,5-c]хіназолінів: окисненням їх гідрованих аналогів або взаємодією [2-(3-арил-1Н-1,2,4-триазол-5-іл)френіл]амінів з хлорангідридами відповідних алкіл(арил)карбонових кислот. Індивідуальність та будова синтезованих сполук підтверджена хроматомас-, ${ }^{1} \mathrm{H}$-, ${ }^{13} \mathrm{C}$-ЯМР-спектрами та елементним аналізом. Показані особливості та відмінності резонування характеристичних протонів 5-R-2-арил-5,6-дигідро-[1,2,4]триазоло[1,5-c]хіназолінів та їх ароматичних аналогів.

\begin{abstract}
5,6-ДИГИДРО-[1,2,4]ТРИАЗОЛО[1,5-С]ХИНАЗОЛИНЫ. СООБЩЕНИЕ 1. ОСОБЕННОСТИ ПОВЕДЕНИЯ [2-(3-АРИЛ-1Н1,2,4-ТРИАЗОЛ-5-ИЛ)ФЕНИЛ]АМИНОВ В РЕАКЦИЯХ С АЛЬДЕГИДАМИ АЛИФАТИЧЕСКОГО И АРОМАТИЧЕСКОГО РЯДА С.В.Холодняк, К.П.Шабельник, А.Ю.Воскобойник, Г.Г.Берест, С.И.Коваленко

Ключевые слова: [2-(3-арил-1Н-1,2,4-триазол-5-ил)фенил]амины; [5+1]-циклоконденсация; спектральные характеристики

Реакции [5+1]-циклоконденсации [2-(3-арил-1Н-1,2,4-триазол-5-ил)френил]аминов с алифатическими и ароматическими альгегидами в зависимости от условий проведения образуют 5-R-2-арил-5, 6-дигидроили 5-R-2-арил-[1,2,4]триазоло[1,5-с]хиназолины. Определены оптимальные условия реакции и установлены основные фракторы, способствующие окислению 5-R-2-арил-5,6-дигидро-[1,2,4]триазоло[1,5-c] хиназолинов. Разработан встречный синтез 5-R-2-арил-[1,2,4]триазоло[1,5-c]хиназолинов: окислением их гидрированных аналогов или взаимодействием [2-(3-арил-1Н-1,2, 4-триазол-5-ил)фенил]аминов с хлорангидридами соответствующих алкил(арил)карбоновых кислот. Индивидуальность и строение синтезированных соединений подтверждены хроматомас-, ${ }^{1} \mathrm{H}$-, ${ }^{13} \mathrm{C}$-ЯМР-спектрами, элементным анализом. Показаны особенности и отличие резонирования характеристических протонов 5-R-2-арил5,6-дигидро-[1,2,4]триазоло[1,5-с]хиназолинов и их ароматических аналогов.
\end{abstract}

Ever-growing interest in quinazoline derivatives $[1,2]$ as potential bioactive agents also attracts attention of the specialists in medicinal chemistry, especially to the condensed derivatives of the heterocyclic system mentioned above, in particular to $[1,2,4]$ triazolo[c]quinazolines. It should be noted that according to the published papers the potential of heteroannelated quinazolines is not limited by their high biologic activity [3-5]. Moreover, these compounds are also the study objects in chemistry of materials $[6,7]$ and chemistry of complex compounds [7]. Among the known methods suitable for the synthesis of the $[1,2,4]$ triazolo[c]quinazoline system, the most used are: domino-reactions that leading to the simultaneous formation of quinazoline and triazole cycles, annulation of the triazole fragment to the quinazoline system and formation of the pyrimidine fragment based on modification of 2-(1H-1,2,4-triazol-5-yl)anilines 
$[1,8]$. It is important that the last-mentioned approach has advantages when it is necessary to vary the nature of a substituent in position 5 and saturation of the pyrimidine fragment. In spite of the fact that methods of the synthesis for $[1,2,4]$ triazolo $[c]$ quinazoline systems via interaction of substituted 2-(1H-1,2,4-triazol-5-yl)anilines with electrophiles are known [7, 9], the objects of most studies published are target compounds and their properties, but not the [5+1]-cyclocondensation process and its peculiarities.

Considering the abovementioned facts we decided to study the interaction of [2-(3-aryl-1H-1,2,4triazole-5-yl)phenyl]amines with carbonyl-containing compounds, as well as to determine the influence of their structure, the nature of solvents and the reaction conditions on the products' structure.

\section{Results and Discussion}

To improve the role of [5+1]-cyclocondensation processes in the synthesis of the triazolo[c]quinazoline system, [2-(3-aryl-1H-1,2,4-triazole-5-yl)phenyl] amines $[10,11]$ as 1,5-binucleophiles were used (1.1-1.9). It was found that interaction of these amines with aromatic and aliphatic aldehydes in the acetic acid medium for 3-6 $\mathrm{h}$ led to formation of the mixture of products. According to spectral data the components of this mixture were 5-R-2-aryl-5,6-dihydro-[1,2,4] triazolo[1,5-c] quinazolines (2) and their aromatic analogues $(3)$ in the ratio of $2: 1$. Further increase in the reaction duration more than $8 \mathrm{~h}$ in most cases led to the quantitative oxidation of the corresponding dihydroderivatives into 2 -aryl-5-R-[1,2,4] triazolo[1,5-c]quinazolines (3.1-3.14) [10]. The most significant factors contributing to oxidation of com- pounds $\mathbf{2}$ are a significant duration of the reaction and high temperature.

The experimental data has shown that compounds 2.1-2.18 may be obtained with excellent yields via refluxing the initial compounds mentioned above for 2-4 $\mathrm{h}$ in alcohols in the presence of an acidic catalyst, as well as with stirring in the same solvent at ambient temperature and under the atmosphere of for $24 \mathrm{~h}$. It should be noted that this reaction may be performed in other solvents that are inert in relation to the initial substances (methanol, propanol-2, propanol-2, dioxane, acetic acid), but the inert atmosphere is the necessary condition.

Considering formation of compounds 2 it proceeded as double nucleophilic addition, wherein the corresponding Schiff base played the role of an intermediate. Further cyclisation progressed as a nonstereoselective transformation and yielded the racemic mixture of 5- $R(S)$-(alkyl-, cycloalkyl-, aryl-)-2-aryl5,6-dihydro[1,2,4] triazolo[1,5-c] quinazolines (2.1-2.18).

For unequivocal evidence of the oxidation process 2-aryl-5-R-[1,2,4] triazolo[1,5-c]quinazolines were prepared according to two alternative protocols. The first approach was based on oxidation of compounds 2.1, 2.2, 2.4, 2.8 and 2.10 with bromine or potassium permanganate (Scheme). Compounds 3.8, 3.9 and $\mathbf{3 . 1 2}$ were obtained via reflux of anilines $\mathbf{1 . 1}$ and 1.8, acylhalides of the corresponding aliphatic and aromatic carboxylic acid and the equimolar quantity of sodium acetate in acetic acid (Scheme). The reaction proceeded as a phased reaction that included acylation followed by cyclisation of the $\mathrm{N}$-acyl derivative formed.

Purity and the structure of the compounds synthesized were confirmed by the complex of physico-
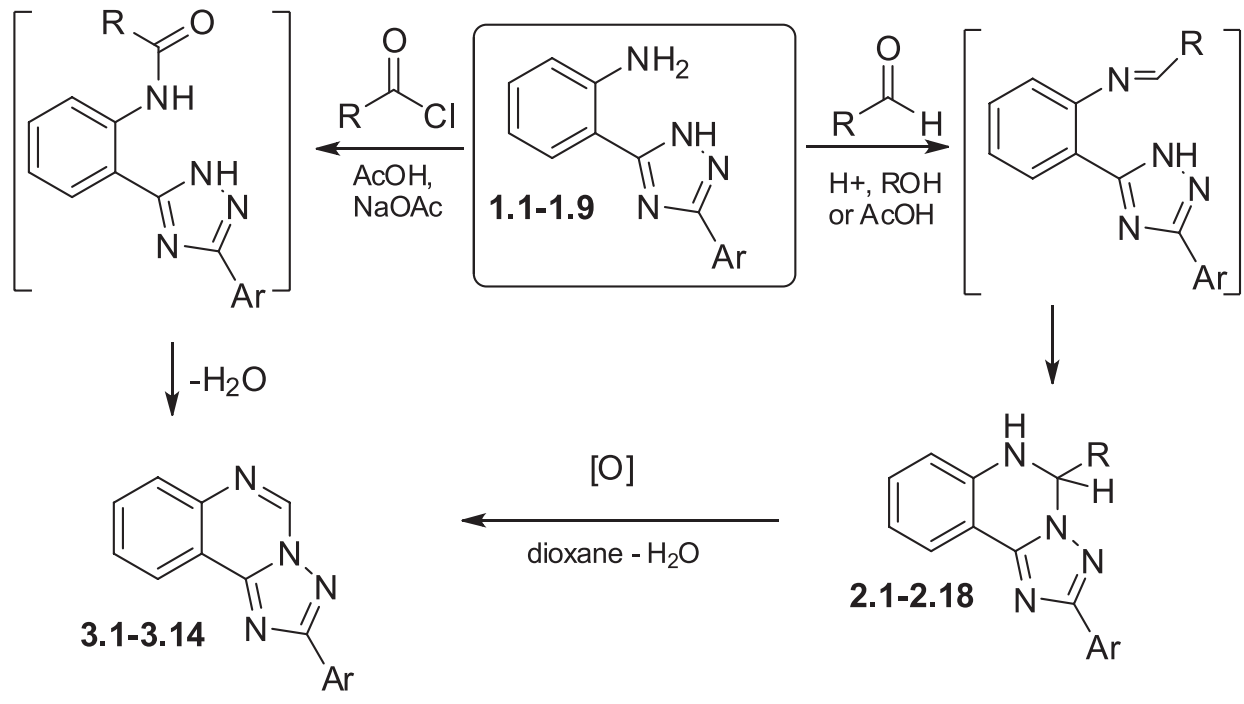

$\mathrm{Ar}=\mathrm{Ph}, 2-\mathrm{CH}_{3} \mathrm{C}_{6} \mathrm{H}_{4}, 3-\mathrm{CH}_{3} \mathrm{C}_{6} \mathrm{H}_{4}, 3-\mathrm{CF}_{3} \mathrm{C}_{6} \mathrm{H}_{4}, 3-\mathrm{FC}_{6} \mathrm{H}_{4}, \quad 4-\mathrm{FC}_{6} \mathrm{H}_{4}, 2-\mathrm{ClC}_{6} \mathrm{H}_{4}$, 3- $\mathrm{CH}_{3} \mathrm{OC}_{6} \mathrm{H}_{4}, 4-\mathrm{CH}_{3} \mathrm{OC}_{6} \mathrm{H}_{4} ; \mathrm{R}=\mathrm{i}-\mathrm{Pr}$, i-Bu, cyclo-Pr, cyclopentyl, cyclohexyl, $\mathrm{Ph}$, 2- $\mathrm{CH}_{3} \mathrm{C}_{6} \mathrm{H}_{4}, \quad 4-\mathrm{CH}_{3} \mathrm{C}_{6} \mathrm{H}_{4}, \quad 2-\mathrm{HOC}_{6} \mathrm{H}_{4}$, 2- $\mathrm{CF}_{3} \mathrm{C}_{6} \mathrm{H}_{4}, \quad 3-\mathrm{CF}_{3} \mathrm{C}_{6} \mathrm{H}_{4}, \quad 4-\mathrm{CF}_{3} \mathrm{C}_{6} \mathrm{H}_{4}$, $2-\mathrm{CIC}_{6} \mathrm{H}_{4}, 3-\mathrm{CIC}_{6} \mathrm{H}_{4}, 4-\mathrm{ClC}_{6} \mathrm{H}_{4}, 2-\mathrm{BrC}_{6} \mathrm{H}_{4}, 3-\mathrm{BrC}_{6} \mathrm{H}_{4}, 4-\mathrm{BrC}_{6} \mathrm{H}_{4}, 2-\mathrm{FC}_{6} \mathrm{H}_{4}, 3-\mathrm{FC}_{6} \mathrm{H}_{4}$, 
chemical methods, including LC-MS, ${ }^{1} \mathrm{H}$ NMR, ${ }^{13} \mathrm{C}$ NMR-, mass-spectrometry and elemental analysis. In LC-MS spectra of all compounds synthesized the high-intensive signals of quasi-molecular ions with the proper molecular weight were detected.

The formation of compounds 2.1-2.18 was proven by the presence of $\mathrm{H}-6$ signals in ${ }^{1} \mathrm{H}$ NMR - spectra at the 7.65-6.90 ppm. For compounds 2.6-2.18 the signals mentioned overlapped with multiplets of the aryl moiety in position 5. Signals of $\mathrm{H}-5$ in ${ }^{1} \mathrm{H}$ NMR spectra of compounds formed as a result of interaction between the initial anilines and aliphatic aldehydes 2.1-2.5 were observed as broad triplets or multiplets at $5.79-5.60 \mathrm{ppm}$. At the same time compounds containing the aromatic moiety in position 5 were characterized by the $\mathrm{H}-5$ singlet at 7.16-6.93 ppm. Additionally ${ }^{1} \mathrm{H}$ NMR-spectra in $\mathrm{CDCl}_{3}$ were registered for compounds $\mathbf{2 . 6}$ and 2.10. It was shown that signals of H-5 and H-6 were observed as singlets at $5.48 \mathrm{ppm}$ and the $4.76 \mathrm{ppm}$, respectively.

The protons of heterocyclic fragments were registered as sequentially located doublets $\mathrm{H}-10$ (7.99$7.72 \mathrm{ppm})$ and $\mathrm{H}-7$ (6.92-6.76 ppm) and triplets of H-8 (7.83-7.16 ppm), H-9 (6.98-6.72 ppm). Signals of substituents at positions 2 and 5 had chemical shifts that were in compliance with their structure [12].

At the same time ${ }^{1} \mathrm{H}$ NMR-spectra of compounds 3.1-3.14 differed significantly from spectra of the reduced analogues. The signals of H-10, H-9, H-8 and $\mathrm{H}-7$ were observed in much more lower field. The signals of $\mathrm{H}-10$ registered at 8.58-8.49 ppm were the most shifted in low field.

In ${ }^{13} \mathrm{C}$ NMR-spectra of compounds 2.10, 3.13, 3.14 the signals of $C 5$ were the most characteristic. Thus, for compound $\mathbf{2 . 1 0}$ the signal mentioned was registered at $67.11 \mathrm{ppm}$, but for $\mathbf{3 . 1 3}$ and $\mathbf{3 . 1 4}$ - at $147.26 \mathrm{ppm}$ and $148.15 \mathrm{ppm}$, respectively, indicating the different hybridization state of carbon.

The spectral characteristics definitely proved the structure of both 6,7-dihydro-[1,2,4]triazolo[1,5-c] quinazoline system and their aromatic analogues.

\section{Experimental Part}

Melting points were determined in open capillary tubes and were uncorrected. The elemental analyses (C, H, N, S) were performed using an ELEMENTAR vario EL Cube analyzer (USA). Analyses were indicated by the symbols of the elements or functions within $\pm 0.3 \%$ of the theoretical values. ${ }^{1} \mathrm{H}$ NMR spectra $(400 \mathrm{MHz})$ and ${ }^{13} \mathrm{C}$ NMR spectra $(100 \mathrm{MHz})$ were recorded on a Varian-Mercury 400 (Varian Inc., Palo Alto, CA, USA) spectrometer with TMS as an internal standard in DMSO-d6 solution. LC-MS were recorded using the chromatography/mass spectrometric system consisting of an "Agilent 1100 Series" high performance liquid chromatograph (Agilent, Palo Alto, CA, USA) equipped with a diode-matrix and an "Agi- lent LC/MSD SL" mass-selective detector (atmospheric pressure chemical ionization - APCI).

Synthetic procedures were conducted according to the commonly used approaches for the synthesis of prospective bioactive agents. All reagents were commercially available ("Sigma-Aldrich", Missouri, USA and "Enamine", Kiev, Ukraine) and used without addition purification. 2-(3-Aryl-1 $H$-1,2,4-triazole-5-yl) phenyl]amines (1.1-1.9) were obtained according to the synthetic protocols described $[10,11]$.

The general method for the synthesis of $5-\mathrm{R}^{1}$ 2 -aryl-5,6-dihydro[1,2,4]triazolo[1,5-c]quinazolines (2.1-2.18). Add $10 \mathrm{mmol}$ of aromatic or aliphatic aldehyde to the solution of $10 \mathrm{mmol}$ of the corresponding $\{2$-[3-aryl-1H-1,2,4-triazolo-5-yl]phenyl\}amine (1.1-1.9) in $10 \mathrm{ml}$ of glacial acetic acid or $10 \mathrm{ml}$ of propanol-2. When using propanol-2 as a solvent add two drops of sulphuric or hydrochloric acid. Reflux the mixture obtained for $2 \mathrm{~h}$ or stir at ambient temperature for $24 \mathrm{~h}$ in the atmosphere of nitrogen. Then cool the mixture and pour into the saturated solution of sodium acetate. Filter the precipitate formed and dry. In case of insufficient purity recrystallize the compounds obtained from methanol.

The compounds (2.1-2.18) synthesized are white crystalline powders, insoluble in water, soluble in alcohols, dioxane and DMF.

5-Isopropyl-2-(3-methoxyphenyl)-5, 6-dihydro [1,2,4]triazolo[1,5-c]quinazoline (2.1). Yield-40.9\%. M.p. $-184-186^{\circ} \mathrm{C} ;{ }^{1}{ }^{\mathrm{H}} \mathrm{NMR}\left(400 \mathrm{MHz}, \mathrm{dmso}_{-} \mathrm{d}_{6}\right) \delta 7.99$ $(\mathrm{d}, J=8.2 \mathrm{~Hz}, 1 \mathrm{H}, \mathrm{H}-10), 7.83(\mathrm{t}, J=7.9 \mathrm{~Hz}, 1 \mathrm{H}, \mathrm{H}-8), 7.32-$ 7.12 (m, 2H, H-5 $\left.\mathrm{PhOCH}_{3}-3, \mathrm{H}-6\right), 7.16$ (m, 2H, H-2,6 $\mathrm{PhOCH}_{3}-3$ ), 6.91 (d, $J=9.9 \mathrm{~Hz}, 1 \mathrm{H}, \mathrm{H}-4 \mathrm{PhOCH}_{3}-3$ ), $6.86(\mathrm{~d}, J=8.0 \mathrm{~Hz}, 1 \mathrm{H}, \mathrm{H}-7), 6.71(\mathrm{t}, J=7.4 \mathrm{~Hz}, 1 \mathrm{H}$, $\mathrm{H}-9$ ), $5.71(\mathrm{~m}, J=3.5 \mathrm{~Hz}, 1 \mathrm{H}, \mathrm{H}-5), 3.88\left(\mathrm{~s}, 3 \mathrm{H},-\mathrm{OCH}_{3}\right.$ ), $2.40\left(\mathrm{~m}, 1 \mathrm{H},-\mathrm{CH}\left(\mathrm{CH}_{3}\right)_{2}\right), 1.03\left(\mathrm{~d}, J=6.9 \mathrm{~Hz}, 3 \mathrm{H},-\mathrm{CH}\left(\mathrm{CH}_{3}\right)_{2}\right)$, $0.90\left(\mathrm{~d}, J=6.8 \mathrm{~Hz}, 3 \mathrm{H},-\mathrm{CH}\left(\mathrm{CH}_{3}\right)_{2}\right)$; LC-MS, $m / z=321$ [M+1], 322 [M+2]; Anal. calcd. for $\mathrm{C}_{19} \mathrm{H}_{20} \mathrm{~N}_{4} \mathrm{O}: \mathrm{C}, 71.23$; H, 6.29; N, 17.49; Found: C, 71.26; H, 6.31; N, 17.52.

5-Isobutyl-2-(3-fluorophenyl)-5,6-dihydro[1,2,4] triazolo[1,5-c]quinazoline (2.2). Yield - 40.9\%. M.p. $129-131^{\circ} \mathrm{C} ;{ }^{1} \mathrm{H}$ NMR (400 MHz, dmso_d $\mathrm{d}_{6}+\mathrm{CCl}_{4}$ ) $\delta 7.92$ (d, $J=7.3 \mathrm{~Hz}, 1 \mathrm{H}, \mathrm{H}-10), 7.79$ (t, $J=7.5 \mathrm{~Hz}, 1 \mathrm{H}, \mathrm{H}-8)$, 7.45 (m, 2H, H-2,6 PhF-3), 7.19 (m, 1H, H-5 PhF-3), $7.11(\mathrm{t}, J=7.8 \mathrm{~Hz}, 1 \mathrm{H}, \mathrm{H}-9), 6.92(\mathrm{~s}, 1 \mathrm{H}, \mathrm{H}-6), 6.87$ (d, $J=7.8 \mathrm{~Hz}, 1 \mathrm{H}, \mathrm{H}-7), 6.78(\mathrm{~m}, 1 \mathrm{H}, \mathrm{H}-4 \mathrm{PhF}-3), 5.79$ (m, 1H, H-5), $2.02\left(\mathrm{~m}, 1 \mathrm{H},-\mathrm{CH}_{2} \mathrm{CH}\left(\mathrm{CH}_{3}\right)_{2}\right), 1.57$ (d, J $\left.=6.0 \mathrm{~Hz}, 1 \mathrm{H},-\mathrm{CH}_{2} \mathrm{CH}\left(\mathrm{CH}_{3}\right)_{2}\right), 1.13(\mathrm{~d}, J=5.3 \mathrm{~Hz}, 6 \mathrm{H}$, $\left.-\mathrm{CH}_{2} \mathrm{CH}\left(\mathrm{CH}_{3}\right)_{2}\right) ; \mathrm{LC}-\mathrm{MS}, m / z=323[\mathrm{M}+1], 324[\mathrm{M}+2]$; Anal. calcd. for $\mathrm{C}_{19} \mathrm{H}_{19} \mathrm{FN}_{4}$ : C, 70.79; $\mathrm{H}, 5.94 ; \mathrm{N}, 17.38$; Found: C, 70.76; H, 5.91; N, 17.33.

5-Cyclopropyl-2-phenyl-5,6-dihydro[1,2,4]triazolo [1,5-c]quinazoline (2.3). Yield - 41.6\%. M.p. - 157$159^{\circ} \mathrm{C} ;{ }^{1} \mathrm{H}$ NMR $\left(400 \mathrm{MHz}, \mathrm{dmso}_{-} \mathrm{d}_{6}+\mathrm{CCl}_{4}\right) \delta 8.11(\mathrm{~d}, J$ $=7.5 \mathrm{~Hz}, 2 \mathrm{H}, \mathrm{H}-2,6 \mathrm{Ph}), 7.87$ (d, J = 7.3 Hz, $1 \mathrm{H}, \mathrm{H}-10$ ), 7.53-7.49 (m, 1H, H-8), 7.49-7.36 (m, 3H, H-3, 5 Ph, H-6), 7.36-7.29 (m, 1H, H-4 Ph), $6.91(\mathrm{t}, J=7.5 \mathrm{~Hz}$, 
1H, H-9), 6.80 (d, $J=7.9 H z, 1 \mathrm{H}, \mathrm{H}-7), 5.60$ (m, $J=$ $5.8 \mathrm{~Hz}, 1 \mathrm{H}, \mathrm{H}-5)$, 3.69-3.60 (m, 1H, H-2 cyclopropyl), 3.60-3.51 (m, 1H, H-3 cyclopropyl), 2.83-2.71 (m, 1H, H-1 cyclopropyl), 2.30-2.13 (m, 2H, H-2, 3 cyclopropyl); LC-MS, $m / z=289$ [M+1], 290 [M+2]; Anal. calcd. for $\mathrm{C}_{18} \mathrm{H}_{16} \mathrm{~N}_{4}$ : C, 74.98; $\mathrm{H}, 5.59 ; \mathrm{N}, 19.43$; Found: C, 75.01; H, 5.62; N, 19.45 .

5-Cyclohexyl-2-phenyl-5,6-dihydro[1,2,4]triazolo [1,5-c]quinazoline (2.4). Yield - 61.8\%. M.p. - 196$198^{\circ} \mathrm{C} ;{ }^{1} \mathrm{H}$ NMR (400 M Hz, dmso_d $+\mathrm{CCl}_{4}$ ) $\delta 8.12(\mathrm{~d}, J$ $=7.5 \mathrm{~Hz}, 2 \mathrm{H}, \mathrm{H}-2,6 \mathrm{Ph}), 7.72(\mathrm{~d}, J=7.8 \mathrm{~Hz}, 1 \mathrm{H}, \mathrm{H}-10)$, 7.48-7.36 (m, 4H, H-3, 4, 5 Ph, H-6), 7.16 (t, $J=7.5 \mathrm{~Hz}$, $1 \mathrm{H}, \mathrm{H}-8), 6.84(\mathrm{t}, J=7.8 \mathrm{~Hz}, 1 \mathrm{H}, \mathrm{H}-9), 6.76(\mathrm{~d}, J=7.9 \mathrm{~Hz}$, 1H, H-7), 5.66 (bs. t, 1H H-5), 2.38 (d, $J=12.7 \mathrm{~Hz}, 2 \mathrm{H}$, $\mathrm{H}-3,5$ cyclohexyl), 2.22 (d, $J=12.7 \mathrm{~Hz}, 2 \mathrm{H}, \mathrm{H}-3,5$ cyclohexyl),), 1.98-1.75 (m, 4H, H-2,6 cyclohexyl), 1.701.75 (m, 1H, H-1 cyclohexyl), 1.24-1.11 (m, 3H, H-4 cyclohexyl); LC-MS, $m / z=331$ [M+1]; Anal. calcd. for $\mathrm{C}_{21} \mathrm{H}_{22} \mathrm{~N}_{4}$ : C, 76.33; H, 6.71; N, 16.96; Found: C, 76.35; $\mathrm{H}, 6.74 ; \mathrm{N}, 17.01$.

2-(3-Methoxyphenyl)-5-cyclohexyl-5,6-dihydro [1,2,4]triazolo[1,5-c]quinazoline (2.5). Yield - 71.3\%. M.p. - $176-177^{\circ} \mathrm{C} ;{ }^{1} \mathrm{H}$ NMR (400 M Hz, dmso_d 6 ) $\delta 7.82$ (d, $J=7.8 \mathrm{~Hz}, 1 \mathrm{H}, \mathrm{H}-10), 7.66$ (d, $J=7.2 \mathrm{~Hz}, 1 \mathrm{H}, \mathrm{H}-6$ $\left.\mathrm{PhOCH}_{3}-3\right), 7.60$ (s, 1H, H-2 $\left.\mathrm{PhOCH}_{3}-3\right), 7.32$ (t, $J=$ $\left.8.1 \mathrm{~Hz}, 1 \mathrm{H}, \mathrm{H}-5 \mathrm{PhOCH}_{3}-3\right), 7.16(\mathrm{t}, J=7.5 \mathrm{~Hz}, 1 \mathrm{H}, \mathrm{H}-8)$, 6.90 (m, 2H, H-6, H-9), 6.85 (d, J = 7.9 Hz, 1H, H-7), 6.72 (t, $J=7.3 \mathrm{~Hz}, 1 \mathrm{H}, \mathrm{H}-9), 5.69$ (m, 1H, H-5), 3.94 $\left(\mathrm{s}, 3 \mathrm{H},-\mathrm{OCH}_{3}\right), 1.71-1.51$ (m, 4H, H-3,5 cyclohexyl), 1.53-1.34 (m, 4H, H-2,6 cyclohexyl), 1.24 (m, 3H, H-1, 4 cyclohexyl); LC-MS, $m / z=361$ [M+1]; Anal. calcd. for $\mathrm{C}_{22} \mathrm{H}_{24} \mathrm{~N}_{4} \mathrm{O}$ : C, 73.31; $\mathrm{H}, 6.71 ; \mathrm{N}, 15.54$; Found: $\mathrm{C}$, 73.34; H, 6.74; N, 15.57.

2,5-Diphenyl-5,6-dihydro-[1,2,4]triazolo[1,5-c]quinazoline (2.6). Yield - 98.7\%. M.p. $-175-177^{\circ} \mathrm{C}$; NMR $\left(400 \mathrm{MHz}, \mathrm{dmso} \mathrm{d}_{6}+\mathrm{CCl}_{4}\right) \delta 8.05(\mathrm{~d}, J=7.3 \mathrm{~Hz}, 2 \mathrm{H}$, $\mathrm{H}-2,6$ 2-Ph), 7.80 (d, J = $7.5 \mathrm{~Hz}, 1 \mathrm{H}, \mathrm{H}-10), 7.65$ (t, 1H, H-4 2-Ph), 7.52 (t, 1H, H-4 5-Ph), 7.47-7.31 (m, 7H, H-3,5 2-Ph, H-2,3,5,6 5-Ph, H-6,), 7.21 (t, J = 7.1 Hz, 1H, $\mathrm{H}-8), 6.93-6.75$ (m, 3H, H-5,7,9); ${ }^{1} \mathrm{H}$ NMR (400 M Hz, $\left.\mathrm{CDCl}_{3}\right) \delta 8.20(\mathrm{~d}, J=6.8 \mathrm{~Hz}, 2 \mathrm{H}, \mathrm{H}-2,6 \mathrm{Ph}), 8.01(\mathrm{~d}, J=$ $7.6 \mathrm{~Hz}, 1 \mathrm{H}, \mathrm{H}-10), 7.53$ (m, 2H, H-4 2-Ph, H-4 5-Ph), 7.45-7.40 (m, 6H, H-3,5 2-Ph, H-2,3,5,6 5-Ph), 7.34 (t, $J=7.4 \mathrm{~Hz}, 1 \mathrm{H}, \mathrm{H}-8), 6.98(\mathrm{t}, J=7.4 \mathrm{~Hz}, 1 \mathrm{H}, \mathrm{H}-9)$, $6.84(\mathrm{~d}, J=7.9 \mathrm{~Hz}, 1 \mathrm{H}, \mathrm{H}-7), 6.35$ (s, 1H, H-5), 5.48 (s, 1H, H-6); LC-MS, $m / z=325$ [M+1]; Anal. calcd. for $\mathrm{C}_{21} \mathrm{H}_{16} \mathrm{~N}_{4}$ : C, 77.76; H, 4.97; N, 17.27; Found: C, 77.73; $\mathrm{H}, 4.94 ; \mathrm{N}, 17.24$.

2-Phenyl-5-(2-hydroxyphenyl)-5,6-dihydro[1,2,4]triazolo[1,5-c]quinazoline (2.7). Yield - 98.2\%. M.p. - $170-172{ }^{\circ} \mathrm{C}$; ${ }^{1} \mathrm{H}$ NMR $\left(400 \mathrm{MHz}, \mathrm{dmso}_{-} \mathrm{d}_{6}+\mathrm{CCl}_{4}\right)$ $\delta 8.01(\mathrm{t}, J=6.3 \mathrm{~Hz}, 1 \mathrm{H}, \mathrm{H}-2,6 \mathrm{Ph}), 7.81(\mathrm{~d}, J=7.3 \mathrm{~Hz}$, 1H, H-10), 7.44-7.30 (m, 3H, H-3,4,5 Ph), 7.30-7.09 (m, 6H, H-3,4,5,6 2-HOPh, H-6, H-8), 7.02 (s, 1H, H-5), 6.85 (d, $J=7.6 \mathrm{~Hz}, 1 \mathrm{H}, \mathrm{H}-7), 6.80$ (t, $7.4 \mathrm{~Hz} 1 \mathrm{H}, \mathrm{H}-9)$, 2.50 (d, $J=1.1 \mathrm{~Hz}, 1 \mathrm{H},-\mathrm{OH})$; LC-MS, $m / z=341$ [M+1]; Anal. calcd. for $\mathrm{C}_{21} \mathrm{H}_{16} \mathrm{~N}_{4} \mathrm{O}$ : C, 74.10; $\mathrm{H}, 4.74 ; \mathrm{N}, 16.46$; Found: C, 74.07; H, 4.70; N, 16.42.
2-Phenyl-5-(2-(trifluoromethyl)phenyl)-5,6-dihydro[1,2,4]triazolo[1,5-c]quinazoline (2.8). Yield - 96.9\%. M.p. - $190-191^{\circ} \mathrm{C} ;{ }^{1} \mathrm{H}$ NMR (400 M Hz, dmso_d $6+\mathrm{CCl}_{4}$ ) $\delta 8.00(\mathrm{~d}, J=7.5 \mathrm{~Hz}, 2 \mathrm{H}, \mathrm{H}-2,6 \mathrm{Ph}), 7.86(\mathrm{~d}, J=7.5 \mathrm{~Hz}$, $1 \mathrm{H}, \mathrm{H}-3$ 2-CF $\mathrm{Ph}$ ), 7.80 (d, J = 7.3 Hz, 1H, H-10), 7.697.53 (m, 3H, H-3,4,5 Ph), 7.36 (m, 4H, H-4,5,6 2-CF ${ }_{3} \mathrm{Ph}$, $\mathrm{H}-6), 7.23$ (t, J = 7.6 Hz, 1H, H-8), 7.11 (s, 1H, H-5), 6.94-6.79 (m, 2H, H-7,9); LC-MS, $m / z=393$ [M+1]; Anal. calcd. for $\mathrm{C}_{22} \mathrm{H}_{15} \mathrm{~F}_{3} \mathrm{~N}_{4}$ : C, 67.34; $\mathrm{H}, 3.85 ; \mathrm{N}, 14.28$; Found: C, 67.36; H, 3.90; N, 14.31.

2-Phenyl-5-(3-(trifluoromethyl)phenyl-5,6-dihydro[1,2,4]triazolo[1,5-c]quinazoline (2.9). Yield - 86.7\%. M.p. $-168-170^{\circ} \mathrm{C} ;{ }^{1} \mathrm{H}$ NMR (400 M Hz, dmso_d $6+\mathrm{CCl}_{4}$ ) $\delta 8.06(\mathrm{~d}, J=6.7 \mathrm{~Hz}, 1 \mathrm{H}, \mathrm{H}-2,6 \mathrm{Ph}), 7.84(\mathrm{~m}, 1 \mathrm{H}, \mathrm{H}-2$ 3-CF $\left.{ }_{3} \mathrm{Ph}, \mathrm{H}-10\right), 7.65$ (s, 1H, H-6), 7.57 (m, 3H, H-4,6 3- $\left.\mathrm{CF}_{3} \mathrm{Ph}, \mathrm{H}-4 \mathrm{Ph}\right), 7.46-7.32\left(\mathrm{~m}, 2 \mathrm{H}, \mathrm{H}-5\right.$ 3- $\mathrm{CF}_{3} \mathrm{Ph}$, $\mathrm{H}-3,5 \mathrm{Ph}), 7.24(\mathrm{t}, J=7.1 \mathrm{~Hz}, 1 \mathrm{H}, \mathrm{H}-8), 7.00$ (s, $1 \mathrm{H}$, H-5), 6.91 (d, $J=7.9 \mathrm{~Hz}, 1 \mathrm{H}, \mathrm{H}-7), 6.84$ (t, $J=7.4 \mathrm{~Hz}$, $1 \mathrm{H}, \mathrm{H}-9)$; LC-MS, $m / z=393$ [M+1]; Anal. calcd. for $\mathrm{C}_{22} \mathrm{H}_{15} \mathrm{~F}_{3} \mathrm{~N}_{4}$ : C, 67.34; H, 3.85; N, 14.28; Found: C, 67.33; $\mathrm{H}, 3.87 ; \mathrm{N}, 14.26$.

2-Phenyl-5-(4-(trifluoromethyl)phenyl)-5,6-dihydro-[1,2,4]triazolo[1,5-c]quinazoline (2.10). Yield-55.1\%. M.p. - $166-168^{\circ} \mathrm{C} ;{ }^{1} \mathrm{H}$ NMR (400 M Hz, dmso_d $+\mathrm{CCl}_{4}$ ) $\delta 8.05(\mathrm{~d}, J=7.0 \mathrm{~Hz}, 2 \mathrm{H}, \mathrm{H}-2,6 \mathrm{Ph}), 7.81(\mathrm{~d}, J=7.6 \mathrm{~Hz}$, $1 \mathrm{H}, \mathrm{H}-10), 7.66$ (d, $\left.J=7.8 \mathrm{~Hz}, 1 \mathrm{H}, \mathrm{H}-2,64-\mathrm{CF}_{3} \mathrm{Ph}\right), 7.62-$ $7.53\left(\mathrm{~m}, J=8.5 \mathrm{~Hz}, 3 \mathrm{H}, \mathrm{H}-3,54-\mathrm{CF}_{3} \mathrm{Ph}, \mathrm{H}-6\right)$, 7.467.32 (m, 3H, H-3,4,5 Ph), 7.23 (t, $J=7.5 \mathrm{~Hz}, 1 \mathrm{H}, \mathrm{H}-8)$, 6.99 (s, 1H, H-5), 6.89 (d, J = $7.9 \mathrm{~Hz}, 1 \mathrm{H}, \mathrm{H}-7), 6.83(\mathrm{t}$, $J=7.4 \mathrm{~Hz}, 1 \mathrm{H}, \mathrm{H}-9) ;{ }^{1} \mathrm{H} \mathrm{NMR}\left(400 \mathrm{MHz}^{\left.-\mathrm{CDCl}_{3}\right) \delta 8.09}\right.$ $(\mathrm{d}, J=6.4 \mathrm{~Hz}, 2 \mathrm{H}, \mathrm{H}-2,6 \mathrm{Ph}), 8.00(\mathrm{~d}, J=7.6 \mathrm{~Hz}, 1 \mathrm{H}$, $\mathrm{H}-10$ ), 7.59 (d, J = 7.7 Hz, 2H, H-2,6 $\mathrm{PhCF}_{3}$ ), 7.49 (d, J $\left.=7.9 \mathrm{~Hz}, 2 \mathrm{H}, \mathrm{H}-3,5 \mathrm{PhCF}_{3}\right), 7.44-7.34(\mathrm{~m}, 3 \mathrm{H}, \mathrm{H}-3,4,5$ $\mathrm{Ph}), 7.27$ (m, 2H, H-8, $\mathrm{CHCl}_{3}$ ), $6.97(\mathrm{t}, J=7.4 \mathrm{~Hz}, 1 \mathrm{H}$, $\mathrm{H}-9$ ), 6.78-6.73 (m, 2H, H-7, H-5), 4.76 (s, 1H, H-6); ${ }^{13} \mathrm{C} \mathrm{NMR}\left(100 \mathrm{MHz} \mathrm{CDCl}_{3}\right.$ ) $\delta 158.12$ (C-2), 145.47 (C-6a), 137.48 (C-1, PhCF3), 136.21 (C-10b), 127.19, 126.01, 124.66, 123.76, 122.46, 121.79, 121.21, 120.32, 115.74, 110.17, 106.80 (C-10a), 67.11 (C-5); LC-MS, $m / z=393[\mathrm{M}+1]$; Anal. calcd. for $\mathrm{C}_{22} \mathrm{H}_{15} \mathrm{~F}_{3} \mathrm{~N}_{4}$ : C, 67.34; H, 3.85; N, 14.28; Found: C, 67.34; H, 3.85; N, 14.28.

2-Phenyl-5-(2-chlorophenyl)-5,6-dihydro-[1,2,4]triazolo[1,5-c]quinazoline (2.11). Yield - 92.7\%. M.p. 204-206 ${ }^{\circ} \mathrm{C} ;{ }^{1} \mathrm{H}$ NMR (400 M Hz, DMSO_D6+CCl4) $\delta$ $8.04(\mathrm{t}, J=7.1 \mathrm{~Hz}, 1 \mathrm{H}, \mathrm{H}-2,6 \mathrm{Ph}), 7.84(\mathrm{~d}, J=7.5 \mathrm{~Hz}$, $1 \mathrm{H}, \mathrm{H}-10), 7.49$ (d, J = 7.8 Hz, 1H, H-3 2-ClPh), 7.457.31 (m, 5H, H-3,4,5 Ph, H-5 2-ClPh, H-6,), 7.28 (t, $J=$ 7.6 Hz, 1H, H-8), 7.25-7.16 (m, 3H, H-4,6 2-ClPh, H-5), 6.92-6.78 (m, 2H, H-7, 9); LC-MS, $m / z=359$ [M+1], 361 [M+3]; Anal. calcd. for $\mathrm{C}_{21} \mathrm{H}_{15} \mathrm{ClN}_{4}$ : C, 70.29; $\mathrm{H}, 4.21$; N, 15.61; Found: C, 70.33; H, 4.24; N, 15.63.

2-Phenyl-5-(3-chlorophenyl)-5,6-dihydro-[1,2,4]triazolo[1,5-c] quinazoline (2.12). Yield - 94.9\%. M.p. $150-152{ }^{\circ} \mathrm{C}$; ${ }^{1} \mathrm{H}$ NMR (400 MHz, dmso_d $6+\mathrm{CCl}_{4}$ ) $\delta 8.07$ (d, $J=7.0 \mathrm{~Hz}, 1 \mathrm{H}, \mathrm{H}-2,6 \mathrm{Ph}$ ), $7.82(\mathrm{~d}, J=7.4 \mathrm{~Hz}, 1 \mathrm{H}$, H-10), 7.54 (s, 1H, H-6), 7.47 (s, 1H, H-2 3-ClPh), 7.457.31 (m, 4H, H-3,5 Ph, H-4,5 3-ClPh), 7.31-7.17 (m, 3H, H-4 Ph, H-6 3-ClPh, H-8), 6.94-6.86 (m, 2H, H-5, 7), 
6.83 (t, J = 7.5 Hz, 1H, H-9); LC-MS, $m / z=359$ [M+1], 361 [M+3]; Anal. calcd. for $\mathrm{C}_{21} \mathrm{H}_{15} \mathrm{ClN}_{4}$ : C, 70.29; $\mathrm{H}, 4.21$; $\mathrm{N}, 15.61$; Found: C, 70.26; H, 4.19; N, 15.59 .

2-Phenyl-5-(4-chlorophenyl)-5,6-dihydro-[1,2,4]triazolo[1,5-cquinazoline (2.13). Yield - 84.3\%. M.p. $172-174^{\circ} \mathrm{C} ;{ }^{1} \mathrm{H}$ NMR (400 MHz, dmso_d $\mathrm{d}_{6}+\mathrm{CCl}_{4}$ ) $\delta 8.05$ (d, $J=7.3 \mathrm{~Hz}, 1 \mathrm{H}, \mathrm{H}-2,6 \mathrm{Ph}$ ), $7.80(\mathrm{~d}, J=7.3 \mathrm{~Hz}, 1 \mathrm{H}$, $\mathrm{H}-10$ ), 7.48 (s, 1H, H-6), 7.46-7.26 (m, 7H, H-2,3,5,6 4-ClPh, H-3,4,5 Ph), 7.22 (t, J = 7.5 Hz, 1H, H-8), 6.93$6.86(\mathrm{~m}, 2 \mathrm{H}, \mathrm{H}-5,7), 6.82(\mathrm{t}, J=7.4 \mathrm{~Hz}, 1 \mathrm{H}, \mathrm{H}-9)$; LC-MS, $m / z=359[\mathrm{M}+1], 361[\mathrm{M}+3]$; Anal. calcd. for $\mathrm{C}_{21} \mathrm{H}_{15} \mathrm{ClN}_{4}$ : C, 70.29; $\mathrm{H}, 4.21 ; \mathrm{N}, 15.61$; Found: $\mathrm{C}$, 70.31; H, 4.23; N, 15.60 .

5-(2-Bromophenyl)-2-phenyl-5,6-dihydro-[1,2,4]triazolo[1,5-c]quinazoline (2.14). Yield - 93.0\%. M.p. 209-210 ${ }^{\circ} \mathrm{C} ;{ }^{1} \mathrm{H}$ NMR (400 MHz, dmso_d $\mathrm{d}_{6}+\mathrm{CCl}_{4}$ ) $\delta 8.04$ (d, $J=6.6 \mathrm{~Hz}, 2 \mathrm{H}, \mathrm{P}-2,6 \mathrm{Ph}), 7.84(\mathrm{~d}, J=7.0 \mathrm{~Hz}, 1 \mathrm{H}$, $\mathrm{H}-10), 7.67$ (d, $J=6.7 \mathrm{~Hz}, 1 \mathrm{H}, \mathrm{H}-3 \mathrm{2}-\mathrm{BrPh}), 7.47-7.16$ (m, 5H, H-6, H-3,4,5 Ph, H-4,5,6 2-BrPh, H-5), 6.956.79 (m, 2H, H-7, 9); LC-MS, $m / z=404$ [M+1]; Anal. calcd. for $\mathrm{C}_{21} \mathrm{H}_{15} \mathrm{BrN}_{4}$ : C, 62.54; $\mathrm{H}, 3.75 ; \mathrm{N}, 13.89$; Found: C, 62.56; H, 3.78; N, 13.90.

5-(3-Bromophenyl)-2-phenyl-5,6-dihydro-[1,2,4]triazolo[1,5-c]quinazoline (2.15). Yield - 84.6\%. M.p. 174-176; LC-MS, $m / z=404$ [M+1]; Anal. calcd. for $\mathrm{C}_{21} \mathrm{H}_{15} \mathrm{BrN}_{4}$ : C, 62.54; $\mathrm{H}, 3.75$; N, 13.89; Found: C, 62.52; H, 3.73; N, 13.87 .

2-(2-Chlorophenyl)-5-(2-fluorophenyl)-5,6-dihydro[1,2,4]triazolo[1,5-c]quinazoline (2.16). Yield - 93.3\%. M.p. $-128-130^{\circ} \mathrm{C} ;{ }^{1} \mathrm{H}$ NMR $\left(400 \mathrm{MHz}, \mathrm{dmso}_{-} \mathrm{d}_{6}+\mathrm{CCl}_{4}\right)$ $\delta 7.89(\mathrm{~m}, 1 \mathrm{H}, \mathrm{H}-62-\mathrm{ClPh}), 7.81(\mathrm{~d}, J=7.2 \mathrm{~Hz}, 1 \mathrm{H}$, $\mathrm{H}-10$ ), 7.51-7.31 (m, 5H, H-3,5 2-FPh, H-3,5 2-ClPh, H-6), 7.29-7.06 (m, 5H, H-4 2-ClPh, H-4,6 2-FPh, H-5, H-8), 6.94-6.76 (m, 2H, H-7, H-9); LC-MS, $m / z=377$ [M+1], 379 [M+3]; Anal. calcd. for $\mathrm{C}_{21} \mathrm{H}_{14} \mathrm{ClFN}_{4}$ : C, 66.94; H, 3.74; N, 14.87; Found: C, 66.92; H, 3.70; N, 14.84 .

2-(2-Chlorophenyl)-5-(2-methylphenyl)-5, 6-dihydro-[1,2,4]triazolo[1,5-c]quinazolines (2.17). Yield 93.9\%. M.p. - 197-199 ${ }^{\circ} \mathrm{C}^{1}{ }^{1} \mathrm{H}$ NMR $(400 \mathrm{MHz}$, dmso_ $\left.\mathrm{d}_{6}+\mathrm{CCl}_{4}\right) \delta$ 7.89-7.82 (m, 1H, H-6 2-ClPh), $7.79(\mathrm{~d}, \bar{J}$ $=7.3 \mathrm{~Hz}, 1 \mathrm{H}, \mathrm{H}-10), 7.49-7.40(\mathrm{~m}, 1 \mathrm{H}, \mathrm{H}-3 \mathrm{2}-\mathrm{ClPh})$, 7.40-7.14 (m, 8H, H-6, H-3,4,5,6 2-- $\mathrm{CH}_{3} \mathrm{Ph}, \mathrm{H}-4,5 \mathrm{CClPh}$, $\mathrm{H}-8$ ), 7.05 (s, 1H, H-5), 6.88 (d, $J=7.9 \mathrm{~Hz}, 1 \mathrm{H}, \mathrm{H}-7$ ), $6.80\left(\mathrm{t}, J=7.2 \mathrm{~Hz}, 1 \mathrm{H}, \mathrm{H}-9\right.$ ), $2.50\left(\mathrm{~s}, 3 \mathrm{H},-\mathrm{CH}_{3}\right.$ ); LC-MS, $m / z=373[\mathrm{M}+1], 375[\mathrm{M}+3]$ Anal. calcd. for $\mathrm{C}_{22} \mathrm{H}_{17} \mathrm{ClN}_{4}$ : C, 70.87; H, 4.60; N, 15.03; Found: C, 70.91; H, 4.63; $\mathrm{N}, 15.05$.

5-(2-Methylphenyl)-2-(3-fluorophenyl)-5,6-dihydro-[1,2,4]triazolo[1,5-c]quinazoline (2.18). Yield-62.1\%. M.p. $-225-227^{\circ} \mathrm{C} ;{ }^{1} \mathrm{H}$ NMR $\left(400 \mathrm{MHz}, \mathrm{dmso}_{-} \mathrm{d}_{6}+\mathrm{CCl}_{4}\right)$ $\delta 7.83(\mathrm{~m}, 2 \mathrm{H}, \mathrm{H}-2,63-\mathrm{FPh}), 7.71(\mathrm{~d}, J=9.4 \mathrm{~Hz}, 1 \mathrm{H}, \mathrm{H}-10)$, 7.53 (m, 1H, H-6), 7.49-7.34 (m, 2H, H-4,5 3-FPh), 7.34-7.14 (m, 2H), 7.14-7.05 (t, 1H, H-8), $7.03(\mathrm{~s}, 1 \mathrm{H}$, $\mathrm{H}-5$ ), $6.86(\mathrm{~d}, J=7.8 \mathrm{~Hz}, 1 \mathrm{H}, \mathrm{H}-7), 6.81(\mathrm{t}, J=7.3 \mathrm{~Hz}$, $1 \mathrm{H}, \mathrm{H}-9), 2.52\left(\mathrm{~s}, 1 \mathrm{H},-\mathrm{CH}_{3}\right.$ ); Anal. calcd. for $\mathrm{C}_{22} \mathrm{H}_{17} \mathrm{FN}_{4}$ : C, 74.14; H, 4.81; N, 15.72; Found: C, 74.16; H, 4.84; $\mathrm{N}, 15.73$.
The general method for the synthesis of $5-\mathrm{R}^{1}$ 2-aryl-[1,2,4]triazolo[1,5-c]quinazolines (3.1-3.14)

Method A. To the solution of $10 \mathrm{mmol}$ of the corresponding $\{2$-[3-aryl-1H-1,2,4-triazol-5-yl]phenyl $\}$ amine (1.1-1.9) in $10 \mathrm{ml}$ of glacial acetic acid add $10 \mathrm{mmol}$ of aliphatic or aromatic aldehyde. Reflux the mixture obtained for $8 \mathrm{~h}$. Then cool the mixture and pour into the saturated solution of sodium acetate. Filter the precipitate formed and dry. In case of insufficient purity recrystallize the compounds obtained from methanol.

Method B. To the solution of $10 \mathrm{mmol}$ of the corresponding 5 - $\mathrm{R}^{1}$-2-aryl-5,6-dihydro[1,2,4]triazolo $[1,5-c]$ quinazoline $(2.2,2.4,2.8,2.10)$ in $10 \mathrm{ml}$ of water - dioxane mixture (1:3) add dropwise $11 \mathrm{mmol}$ of bromine or potassium permanganate at $40^{\circ} \mathrm{C}$. Reflux the mixture obtained for $8 \mathrm{~h}$. Then cool the mixture and pour into the saturated solution of sodium acetate. Filter the precipitate formed and dry. In case of insufficient purity recrystallize the compounds obtained from propanol-1.

Method C. To the solution of $10 \mathrm{mmol}$ of $\{2-[3-$ phenyl-1H-1,2,4-triazol-5-yl]phenyl \}amine (5.1) in $10 \mathrm{ml}$ of glacial acetic acid add $0.9 \mathrm{~g}$. (11 mmol) of sodium acetate. Cool the mixture obtained to $3-5^{\circ} \mathrm{C}$ and add dropwise $11 \mathrm{mmol}$ of the corresponding acyl halide while stirring. Then reflux the reaction mixture for $6 \mathrm{~h}$. After refluxing cool the mixture, and evaporate the solvent under vacuum. Then add $10 \mathrm{ml}$ of methanol, filter the precipitate and dry. Compounds 3.1-3.14 that were obtained according to methods A, B, C had the same physicochemical constants and spectral characteristics.

The compounds (3.1-3.14) synthesized are white crystalline powders, insoluble in water, soluble in alcohols, dioxane and DMF.

5-Isopropyl-2-(4-methoxyphenyl)-[1,2,4]triazolo [1,5-c]quinazoline (3.1). Yield- 48.7 (Method A), 86.8\% (Method B). M.p. $-150-152^{\circ} \mathrm{C} ;{ }^{1} \mathrm{H}$ NMR $(400 \mathrm{MHz}$, dmso_d $\left.{ }_{6}+\mathrm{CCl}_{4}\right) \delta 8.50(\mathrm{~d}, J=7.9 \mathrm{~Hz}, 1 \mathrm{H}, \mathrm{H}-10), 8.24$ $\left(\mathrm{d}, J=6.9 \mathrm{~Hz}, 2 \mathrm{H}, \mathrm{H}-2,6 \mathrm{PhOCH}_{3}-4\right), 8.01(\mathrm{~d}, J=7.8 \mathrm{~Hz}$, $1 \mathrm{H}, \mathrm{H}-7$ ), 7.83 (t, 1H, H-9), 7.73 (d, $J=7.6 \mathrm{~Hz}, 1 \mathrm{H}, \mathrm{H}-8$ ), $7.04\left(\mathrm{~d}, J=7.0 \mathrm{~Hz}, 2 \mathrm{H}, \mathrm{H}-3,5 \mathrm{PhOCH}_{3}-4\right.$ ), 4.04 (dt, $\left.J=14.7,7.4 \mathrm{~Hz}, 1 \mathrm{H},\left(\mathrm{CH}_{3}\right)_{2} \mathrm{C}-\mathrm{H}-\right), 3.89\left(\mathrm{~s}, 1 \mathrm{H}, \mathrm{OCH}_{3}\right)$, 1.56 (s, 6H, $\left.\left(\mathrm{CH}_{3}\right)_{2} \mathrm{CH}-\right)$; LC-MS, $m / z=319[\mathrm{M}+1]$; Anal. calcd. for $\mathrm{C}_{19} \mathrm{H}_{18} \mathrm{~N}_{4} \mathrm{O}: \mathrm{C}, 71.68 ; \mathrm{H}, 5.70 ; \mathrm{N}, 17.69$; Found: C, 71.69; H, 5.75; N, 17.67.

5-Isobutyl-2-(3-fluorophenyl)-[1,2,4]triazolo[1,5-c] quinazoline (3.2). Yield - 60.3\% (Method A), 91.8\% (Method Б). M.p. $-129-131^{\circ} \mathrm{C} ;{ }^{1} \mathrm{H}$ NMR $(400 \mathrm{MHz}$, dmso_d $\left.\mathrm{d}_{6}+\mathrm{CCl}_{4}\right) \delta 8.50(\mathrm{~d}, J=7.6 \mathrm{~Hz}, 1 \mathrm{H}, \mathrm{H}-10), 8.15$ (d, $J=7.6 \mathrm{~Hz}, 1 \mathrm{H}, \mathrm{H}-7), 8.03-7.97(\mathrm{~m}, 2 \mathrm{H}, \mathrm{H}-2,6 \mathrm{PhF}-$ 3), $7.87(\mathrm{~m}, 1 \mathrm{H}, \mathrm{H}-9), 7.75(\mathrm{~m}, 1 \mathrm{H}, \mathrm{H}-8), 7.56(\mathrm{~m}, 1 \mathrm{H}$, $\mathrm{H}-5 \mathrm{PhF}-3), 7.26$ (t, $J=8.1 \mathrm{~Hz}, 1 \mathrm{H}, \mathrm{H}-4 \mathrm{PhF}-3), 3.27$ $\left(\mathrm{d}, J=6.0 \mathrm{~Hz}, 1 \mathrm{H},-\mathrm{C}_{2} \mathrm{CH}\left(\mathrm{CH}_{3}\right)_{2}\right), 2.69-2.56(\mathrm{~m}, 1 \mathrm{H}$, $\left.-\mathrm{CH}_{2} \mathrm{CH}\left(\mathrm{CH}_{3}\right)_{2}\right), 1.14\left(\mathrm{~s}, J=5.3 \mathrm{~Hz}, 6 \mathrm{H},-\mathrm{CH}_{2} \mathrm{CH}\left(\mathrm{CH}_{3}\right)_{2}\right)$; LC-MS, $m / z=321[\mathrm{M}+1], 323[\mathrm{M}+3]$; Anal. calcd. 
for $\mathrm{C}_{19} \mathrm{H}_{17} \mathrm{FN}_{4}$ : $\mathrm{C}, 71.23 ; \mathrm{H}, 5.35 ; \mathrm{N}, 17.49$; Found: $\mathrm{C}$, 71.20; H, 5.31; N, 17.46.

5-Cyclopentyl-2-(4-fluorophenyl)-[1,2,4]triazolo [1,5-c]quinazoline (3.3). Yield - 39.5\% (Method A). M.p. $-196-198^{\circ} \mathrm{C} ;{ }^{1} \mathrm{H}$ NMR $\left(400 \mathrm{MHz}, \mathrm{dmso}_{-} \mathrm{d}_{6}+\mathrm{CCl}_{4}\right)$ $\delta 8.49(\mathrm{~d}, J=7.7 \mathrm{~Hz}, 1 \mathrm{H}, \mathrm{H}-10), 8.40-8.31(\mathrm{~m}, 2 \mathrm{H}$, H-2,6 PhF-4), 7.96 (d, J = 7.7 Hz, 1H, H-7), 7.87 (t, J $=7.3 \mathrm{~Hz}, 1 \mathrm{H}, \mathrm{H}-9), 7.72(\mathrm{t}, J=6.9 \mathrm{~Hz}, 1 \mathrm{H}, \mathrm{H}-8), 7.33-$ $7.22(\mathrm{~m}, 2 \mathrm{H}, \mathrm{H}-3,5 \mathrm{PhF}-4), 4.13(\mathrm{dt}, J=13.7,6.7 \mathrm{~Hz}$, $1 \mathrm{H}, \mathrm{H}-1$ cyclopenthyl), $2.32(\mathrm{dd}, J=17.0,9.4 \mathrm{~Hz}, 2 \mathrm{H}$, H-2,5 cyclopenthyl), 2.23-2.11 (m, 2H, H-2,5 cyclopenthyl), 2.02-1.88 (m, 2H, H-3,4 cyclopenthyl), 1.83 (dd, $J=13.7,12.0 \mathrm{~Hz}, 2 \mathrm{H}, \mathrm{H}-3,4$ cyclopenthyl); LC-MS, $\mathrm{m} / \mathrm{z}=333$ [M+1], 335 [M+3]; Anal. calcd. for $\mathrm{C}_{20} \mathrm{H}_{17} \mathrm{FN}_{4}$ : C, 72.27; H, 5.16; N, 16.86; Found: C, 72.31; H, 5.19; $\mathrm{N}, 16.88$.

2-(3-(Trifluoromethyl)phenyl)-5-cyclopentyl$[1,2,4]$ triazolo[1,5-c]quinazoline (3.4). Yield - 49.9\% (Method A). M.p. $-138-140^{\circ} \mathrm{C} ;{ }^{1} \mathrm{H}$ NMR $(400 \mathrm{MHz}$, dmso_d $\left.+\mathrm{CCl}_{4}\right) \delta 8.58\left(\mathrm{~m}, 2 \mathrm{H}, \mathrm{H}-2,6 \mathrm{PhCF}_{3}-3\right), 8.53(\mathrm{~d}$, $J=8.0 \mathrm{~Hz}, 1 \mathrm{H}, \mathrm{H}-10), 7.98(\mathrm{~d}, J=8.0 \mathrm{~Hz}, 1 \mathrm{H}, \mathrm{H}-7), 7.87$ (t, 1H, H-9), 7.84-7.71 (m, 3H, H-8, H-4, $5 \mathrm{PhCF}_{3}-3$ ), 4.22 (qun, $J=15.9,7.8 \mathrm{~Hz}, 1 \mathrm{H}, \mathrm{H}-1$ cyclopenthyl), 2.392.25 (m, 2H, H-2,5 cyclopenthyl), $2.25-2.12$ (m, 2H, $\mathrm{H}-2,5$ cyclopenthyl), 2.02-1.90 (m, 2H, H-3,4 cyclopenthyl), 1.90-1.79 (m, 2H, H-3,4 cyclopenthyl); Anal. calcd. for $\mathrm{C}_{21} \mathrm{H}_{17} \mathrm{~F}_{3} \mathrm{~N}_{4}$ : C, 65.96; $\mathrm{H}, 4.48 ; \mathrm{N}, 14.65$; Found: C, 66.01; H, 4.53; N, 14.68.

2-(3-Methoxyphenyl)-5-cyclopentyl-[1,2,4]triazolo [1,5-c]quinazoline (3.5). Yield - 70.1\% (Method A). M.p. $-149-151^{\circ} \mathrm{C} ;{ }^{1} \mathrm{H}$ NMR $\left(400 \mathrm{MHz}\right.$, dmso_d $\left._{6}\right) \delta$ 8.52 (d, $J=7.8 \mathrm{~Hz}, 1 \mathrm{H}, \mathrm{H}-10), 7.96$ (d, $J=8.2 \mathrm{~Hz}, 1 \mathrm{H}$, H-6 $\mathrm{PhOCH}_{3}-3$ ), 7.90 (d, J = 7.6 Hz, 1H, H-7), 7.88-7.79 (m, 2H, H-2, $\left.5 \mathrm{PhOCH}_{3}-3\right), 7.72(\mathrm{t}, J=7.5 \mathrm{~Hz}, 1 \mathrm{H}, \mathrm{H}-9$ ), $7.42(\mathrm{t}, J=7.9 \mathrm{~Hz}, 1 \mathrm{H}, \mathrm{H}-8), 7.04(\mathrm{~d}, J=8.0 \mathrm{~Hz}, 1 \mathrm{H}$, $\mathrm{H}-4 \mathrm{PhOCH}_{3}-3$ ), 4.22-4.08 (m, 1H, H-1 cyclopenthyl), $3.93\left(\mathrm{~s}, 3 \mathrm{H}, \mathrm{OCH}_{3}\right), 2.31(\mathrm{dt}, J=11.9,8.1 \mathrm{~Hz}, 2 \mathrm{H}, \mathrm{H}-2,5$ cyclopenthyl), $2.19(\mathrm{dt}, J=20.2,7.3 \mathrm{~Hz}, 2 \mathrm{H}, \mathrm{H}-2,5 \mathrm{cy}-$ clopenthyl), 2.01-1.90 (m, 2H, H-3,4 cyclopenthyl), 1.90-1.76 (m, 2H, H-3,4 cyclopenthyl); LC-MS, $m / z=$ $345[\mathrm{M}+1]$, 347 [M+3]; Anal. calcd. for $\mathrm{C}_{21} \mathrm{H}_{20} \mathrm{~N}_{4} \mathrm{O}$ : C, 73.23; H, 5.85; N, 16.27; Found: C, 73.20; H, 5.83; N, 16.24 .

5-Cyclohexyl-2-phenyl-[1,2,4]triazolo[1,5-c]quinazoline (3.6). Yield - 85.4\% (Method B). M.p. $-196-198^{\circ} \mathrm{C}$; ${ }^{1} \mathrm{H}$ NMR $\left(400 \mathrm{MHz}\right.$, dmso_d $\left.{ }_{6}+\mathrm{CCl}_{4}\right) \delta 8.51(\mathrm{~d}, J=7.8 \mathrm{~Hz}$, $1 \mathrm{H}, \mathrm{H}-10), 8.34$ (d, J = 6.5 Hz, 2H, H-2,6 Ph), 7.96 (d, $J=7.8 \mathrm{~Hz}, 1 \mathrm{H}, \mathrm{H}-7), 7.85(\mathrm{t}, J=7.3 \mathrm{~Hz}, 1 \mathrm{H}, \mathrm{H}-9), 7.72$ $(\mathrm{t}, J=7.0 \mathrm{~Hz}, 1 \mathrm{H}, \mathrm{H}-8), 7.53(\mathrm{~m}, 3 \mathrm{H}, \mathrm{H}-3,4,5 \mathrm{Ph}), 3.77$ (t, 1H, H-1 cyclohexyl), 2.22 (d, $J=12.7 \mathrm{~Hz}, 2 \mathrm{H}, \mathrm{H}-2$, 6 cyclohexyl), 2.00 (d, 2H, H-2, 6 cyclohexyl), 1.93-1.78 (m, 4H, H-3, 5 cyclohexyl), 1.70-1.55 (m, 2H, H-4 cyclohexyl) ; LC-MS, $m / z=329$ [M+1], 331 [M+3]; Anal. calcd. for $\mathrm{C}_{21} \mathrm{H}_{20} \mathrm{~N}_{4}$ : C, 76.80; H, 6.14; N, 17.06; Found: C, 76.78; H, 6.12; N, 17.04.

2-(3-Methoxyphenyl)-5-cyclohexyl-[1,2,4]triazolo [1,5-c]quinazoline (3.7). Yield - 43.0\% (Method A). M.p. $-176-177^{\circ} \mathrm{C} ;{ }^{1} \mathrm{H}$ NMR (400 $\mathrm{MHz}, \mathrm{dmso}_{-} \mathrm{d}_{6}$ ) $\delta$ $8.52(\mathrm{~d}, J=7.8 \mathrm{~Hz}, 1 \mathrm{H}, \mathrm{H}-10), 7.96(\mathrm{~d}, J=8.1 \mathrm{~Hz}, 1 \mathrm{H}$, $\mathrm{H}-7$ ), 7.90 (d, $J=7.5 \mathrm{~Hz}, 1 \mathrm{H}, \mathrm{H}-6 \mathrm{PhOCH}_{3}-3$ ), 7.887.77 (m, 2H, H-9, H-2 $\left.\mathrm{PhOCH}_{3}-3\right), 7.72(\mathrm{t}, J=7.4 \mathrm{~Hz}$, $1 \mathrm{H}, \mathrm{H}-8), 7.42\left(\mathrm{t}, J=7.9 \mathrm{~Hz}, 1 \mathrm{H}, \mathrm{H}-5 \mathrm{PhOCH}_{3}-3\right), 7.04$ (d, J = $\left.8.0 \mathrm{~Hz}, 1 \mathrm{H}, \mathrm{H}-4 \mathrm{PhOCH}_{3}-3\right), 3.94\left(\mathrm{~s}, 3 \mathrm{H},-\mathrm{OCH}_{3}\right)$, $3.76(\mathrm{t}, J=8.0 \mathrm{~Hz}, 1 \mathrm{H}, \mathrm{H}-1$ cyclohexyl), 2.22 (dd, $J=$ 12.0, 2H, H-2, 6 cyclohexyl), 1.99 (d, 2H, H-2, 6 cyclohexyl), 1.84-1.80 (m, 2H, H-3, 5 cyclohexyl), 1.711.51 (m, 2H, H-3, 5 cyclohexyl), 1.42 (m, 2H, H-4 cyclohexyl) ; LC-MS, $m / z=359$ [M+1], 361 [M+3]; Anal. calcd. for $\mathrm{C}_{22} \mathrm{H}_{22} \mathrm{~N}_{4} \mathrm{O}: \mathrm{C}, 73.72 ; \mathrm{H}, 6.19 ; \mathrm{N}, 15.63$; Found: C, 73.74; H, 6.21; N, 15.65 .

5-(4-Methylphenyl)-2-phenyl-[1,2,4]triazolo[1,5-c] quinazoline (3.8). Yield - 94.6\% (Method A), 67.8\% (Method C). M.p. $-183-185^{\circ} \mathrm{C} ;{ }^{1} \mathrm{H}$ NMR $(400 \mathrm{MHz}$, dmso_d $\left.{ }_{6}+\mathrm{CCl}_{4}\right) \delta$ 8.61-8.51 (m, 3H, H-2,6 $\mathrm{PhCH}_{3}-4$, $\mathrm{H}-10$ ), 8.33 (d, $J=6.1 \mathrm{~Hz}, 2 \mathrm{H}, \mathrm{H}-2,6 \mathrm{Ph}$ ), 8.05 (d, $J=$ $7.9 \mathrm{~Hz}, 1 \mathrm{H}, \mathrm{H}-7), 7.88(\mathrm{t}, J=7.1 \mathrm{~Hz}, 1 \mathrm{H}, \mathrm{H}-9), 7.75(\mathrm{t}$, $J=7.1 \mathrm{~Hz}, 1 \mathrm{H}, \mathrm{H}-8), 7.52(\mathrm{~m}, 3 \mathrm{H}, \mathrm{H}-3,4,5 \mathrm{Ph}), 7.41$ (d, J= 7.4 Hz, 2H, H-3,5 $\left.\mathrm{PhCH}_{3}-4\right), 2.50\left(\mathrm{~s}, 1 \mathrm{H},-\mathrm{CH}_{3}\right)$; LC-MS, $m / z=337$ [M+1], 339 [M+3]; Anal. calcd. for $\mathrm{C}_{22} \mathrm{H}_{16} \mathrm{~N}_{4}$ : C, 78.55; $\mathrm{H}, 4.79$; N, 16.66; Found: C, 78.56; $\mathrm{H}, 4.81 ; \mathrm{N}, 16.67$.

5-(4-Bromophenyl)-2-phenyl-[1,2,4]triazolo[1,5-c] quinazoline (3.9). Yield - 41.8\% (Method A), 78.3\% (Method C). M.p. $-217-219^{\circ} \mathrm{C} ;{ }^{1} \mathrm{H}$ NMR $(400 \mathrm{MHz}$, dmso_d $\left.\mathrm{d}_{6}+\mathrm{CCl}_{4}\right) \delta 8.65(\mathrm{~d}, J=7.8 \mathrm{~Hz}, 2 \mathrm{H}, \mathrm{H}-2,6 \mathrm{Ph})$, $8.59(\mathrm{~d}, J=7.8 \mathrm{~Hz}, 1 \mathrm{H}, \mathrm{H}-10), 8.35(\mathrm{~d}, J=7.0 \mathrm{~Hz}, 2 \mathrm{H}$, $\mathrm{H}-2,6 \mathrm{PhBr}-4), 8.10$ (d, $J=7.8 \mathrm{~Hz}, 1 \mathrm{H}, \mathrm{H}-7$ ), 7.92 (t, $J=7.7 \mathrm{~Hz}, 1 \mathrm{H}, \mathrm{H}-9), 7.85-7.77(\mathrm{~m}, 3 \mathrm{H}, \mathrm{H}-8, \mathrm{H}-3,5$ PhBr-4), 7.60-7.48 (m, 3H, H-3,4,5 Ph); Anal. calcd. for $\mathrm{C}_{21} \mathrm{H}_{13} \mathrm{BrN}_{4}$ : C, 62.86; $\mathrm{H}, 3.27$; N, 13.96; Found: C, 62.88; H, 3.31; N, 14.01 .

2-(3-(Trifluoromethyl)phenyl)-5-(3-fluorophenyl)[1,2,4]triazolo[1,5-c]quinazoline (3.10). Yield - 39.7\% (Method A). M.p. $-148-150^{\circ} \mathrm{C} ;{ }^{1} \mathrm{H}$ NMR $(400 \mathrm{MHz}$, dmso_d $\left.{ }_{6}+\mathrm{CCl}_{4}\right) \delta$ 8.65-8.50 (m, 5H, H-2,6 PhF-3, $\left.\mathrm{H}-2,6 \mathrm{PhCF}_{3}-3, \mathrm{H}-10\right), 8.42$ (d, $J=7.8 \mathrm{~Hz}, 1 \mathrm{H}, \mathrm{H}-7$ ), $8.10(\mathrm{~m}, 1 \mathrm{H}, \mathrm{H}-9), 7.94$ (dd, $J=15.6,7.8 \mathrm{~Hz}, 1 \mathrm{H}, \mathrm{H}-5$ $\mathrm{PhCF}_{3}-3$ ), 7.88-7.73 (m, 2H, H-8, H-4 $\mathrm{PhCF}_{3}-3$ ), 7.68 (dd, $J=14.6,7.2 \mathrm{~Hz}, 1 \mathrm{H}, \mathrm{H}-5, \mathrm{PhF}-3), 7.44(\mathrm{t}, J=7.9 \mathrm{~Hz}$, 1H, H-4 PhF-3); LC-MS, $m / z=409$ [M+1], 411 [M+3]; Anal. calcd. for $\mathrm{C}_{22} \mathrm{H}_{12} \mathrm{~F}_{4} \mathrm{~N}_{4}$ : C, 64.71; $\mathrm{H}, 2.96 ; \mathrm{N}, 13.72$; Found: C, 64.72; H, 2.99; N, 13.75.

2-(3-Methylphenyl)-5-(3-fluorophenyl)-[1,2,4]triazolo[1,5-c]quinazoline (3.11). Yield - 41.6\% (Method A). M.p. $-141-143^{\circ} \mathrm{C} ;{ }^{1} \mathrm{H}$ NMR ( $400 \mathrm{MHz}$, dmso $\left.\mathrm{d}_{6}+\mathrm{CCl}_{4}\right) \delta 8.99\left(\mathrm{~m}, 2 \mathrm{H}, \mathrm{H}-2 \mathrm{PhCF}_{3}-3, \mathrm{H}-10\right), 8.60(\mathrm{~d}, J=$ $7.3 \mathrm{~Hz}, 1 \mathrm{H}, \mathrm{H}-7$ ), 8.22 (d, J = 7.5 Hz, 2H, H-4, $6 \mathrm{PhCF}_{3}-3$ ), $8.14\left(\mathrm{~d}, J=7.8 \mathrm{~Hz}, 1 \mathrm{H}, \mathrm{H}-6 \mathrm{PhCH}_{3}-3\right), 8.03-7.78(\mathrm{~m}$, $\left.3 \mathrm{H}, \mathrm{H}-9, \mathrm{H}-5 \mathrm{PhCF}_{3}-3, \mathrm{H}-5 \mathrm{PhCH}_{3}-3\right), 7.35$ (m, 2H, H-8, $\mathrm{H}-4 \mathrm{PhCH}_{3}-3$ ), 2.47 (s, 2H) ; LC-MS, $m / z=405$ [M+1], 406 [M+2]; Anal. calcd. for $\mathrm{C}_{23} \mathrm{H}_{15} \mathrm{~F}_{3} \mathrm{~N}_{4}$ : C, 68.31; $\mathrm{H}$, 3.74; N, 13.85; Found: C, 68.29; H, 3.72; N, 13.81.

2-(3-Methoxyphenyl)-5-(4-chlorophenyl)-[1,2,4]triazolo[1,5-c]quinazoline (3.12). Yield - 37.9\% (Method A), $61.6 \%$ (Method C). M.p. - 217-219 C; ${ }^{1} \mathrm{H}$ NMR (400 MHz, 
dmso_d $\left.{ }_{6}+\mathrm{CCl}_{4}\right) \delta 8.68(\mathrm{~d}, J=8.0 \mathrm{~Hz}, 2 \mathrm{H}, \mathrm{H}-2,6 \mathrm{PhCl}-$ 4), $8.57(\mathrm{~d}, J=7.7 \mathrm{~Hz}, 1 \mathrm{H}, \mathrm{H}-10), 8.07$ (d, $J=8.0 \mathrm{~Hz}$, 1H, H-7), 7.91-7.87 (m, 2H, H-9, H-6 $\left.\mathrm{PhOCH}_{3}-3\right)$, 7.85-7.76 (m, 2H, H-8, H-2 $\left.\mathrm{PhOCH}_{3}-3\right), 7.64(\mathrm{~d}, J=$ $8.2 \mathrm{~Hz}, 2 \mathrm{H}, \mathrm{H}-3,5 \mathrm{PhCl}-4), 7.47$ (t, J = 7.4 Hz, 1H, H-5 $\mathrm{PhOCH}_{3}-3$ ), 7.04 (d, $J=7.4 \mathrm{~Hz}, 1 \mathrm{H}, \mathrm{H}-4 \mathrm{PhOCH}_{3}-3$ ), 3.92 (s, 3H, $-\mathrm{OCH}_{3}$ ); LC-MS, $m / z=387[\mathrm{M}+1], 389$ [M+3]; Anal. calcd. for $\mathrm{C}_{22} \mathrm{H}_{15} \mathrm{ClN}_{4} \mathrm{O}: \mathrm{C}, 68.31 ; \mathrm{H}, 3.91$; N, 14.48; Found: C, 68.34; H, 3.93; N, 14.51 .

2-Phenyl-5-(2-(trifluoromethyl)phenyl)-[1,2,4]triazolo[1,5-c]quinazoline (3.13). Yield - 43.8\% (Method B). M.p. - $192-194^{\circ} \mathrm{C} ;{ }^{1} \mathrm{H}$ NMR $\left(400 \mathrm{MHz}, \mathrm{CDCl}_{3}\right) \delta 8.66$ (d, J = 7.9 Hz, 1H, H-10), 8.26 (н.p. d, 2H, H-2,6 Ph), 8.10 (d, $J=8.2 \mathrm{~Hz}, 1 \mathrm{H}, \mathrm{H}-7$ ), 7.94-7.83 (m, 2H, H-9, $\left.\mathrm{H}-6 \mathrm{PhCF}_{3}-2\right), 7.82-7.71$ (m, 4H, H-8, H-3,4,5 $\mathrm{PhCF}_{3}-2$ ), 7.48-7.36 (m, 3H, H-3,4,5 Ph); ${ }^{13} \mathrm{C}$ NMR $(100 \mathrm{MHz}$, $\mathrm{CDCl}_{3}$ ) $\delta 159.60$ (C-2), 147.26 (C-5), 137.95 (C-6a), 127.39 (C-10b), 127.08, 126.26, 125.95, 125.65, 125.33, $124.16,124.14,123.87,123.71,122.93,122.39,121.85$, 120.22, 119.10, 112.93 (C-10a); Anal. calcd. for $\mathrm{C}_{22} \mathrm{H}_{13} \mathrm{~F}_{3} \mathrm{~N}_{4}$ : C, 67.69; H, 3.36; N, 14.35; Found: C, 67.71; H, 3.39; $\mathrm{N}, 14.36$.

2-Phenyl-5-(4-(trifluoromethyl)phenyl)-[1,2,4]triazolo[1,5-c]quinazoline (3.14). Yield - 57.3\% (Method B). M.p. - $205-207^{\circ} \mathrm{C} ;{ }^{1} \mathrm{H}$ NMR $\left(400 \mathrm{MHz}, \mathrm{CDCl}_{3}\right) \delta 8.80$ (d, $\left.J=8.1 \mathrm{~Hz}, 2 \mathrm{H}, \mathrm{H}-2,6 \mathrm{PhCF}_{3}-4\right), 8.64$ (d, $J=7.9 \mathrm{~Hz}$,
1H, H-10), 8.38 (d, J = 5.8 Hz, 1H, H-2, $6 \mathrm{Ph}$ ), 8.12 (d, $J=8.2 \mathrm{~Hz}, 1 \mathrm{H}, \mathrm{H}-7), 7.93-7.82\left(\mathrm{~m}, 3 \mathrm{H}, \mathrm{H}-3,5 \mathrm{PhCF}_{3}-4\right.$, $\mathrm{H}-9), 7.75(\mathrm{t}, J=7.5 \mathrm{~Hz}, 1 \mathrm{H}, \mathrm{H}-8), 7.59-7.46(\mathrm{~m}, 3 \mathrm{H}$, $\mathrm{H}-3,4,5 \mathrm{Ph}) ;{ }^{13} \mathrm{C}$ NMR (100 MHz, $\mathrm{CDCl}_{3}$ ) $\delta 159.48$ (C-2), 148.14 (C-5), 140.11 (C-6a), 137.95 (C-10b), 130.34, $127.47,126.12,125.87,125.27,124.14,124.03,124.00$, 122.90, 120.57, 120.53, 120.50, 119.10, 112.77 (C-10a); LC-MS, $m / z=391[\mathrm{M}+1]$, 393 [M+3]; Anal. calcd. for $\mathrm{C}_{22} \mathrm{H}_{13} \mathrm{~F}_{3} \mathrm{~N}_{4}$ : C, 67.69; $\mathrm{H}, 3.36$; $\mathrm{N}, 14.35$; Found: $\mathrm{C}$, 67.68; H, 3.35; N, 14.35 .

\section{Conclusions}

The reaction of [2-(3-aryl-1H-1,2,4-triazole-5-yl) phenyl]amines with aliphatic and aromatic aldehydes is a suitable method for the synthesis of 5-(alkyl-, cycloalkyl-, aryl-)-2-aryl-5,6-dihydro[1,2,4]triazolo [1,5-c]quinazolines. These compounds are oxidizable; therefore, the inert gas atmosphere is the necessary condition for the [5+1]-cyclocondensation process. On the other hand, this property allows to use 5- (alkyl-, cycloalkyl-, aryl-)-2-aryl-5,6-dihydro[1,2,4]triazolo[1,5-c] quinazolines as the initial compounds for the synthesis of their aromatic analogues. The convenient synthetic procedures for compounds $\mathbf{2}$ and 3 have been proposed, and the mechanism of these transformations has been discussed.

\section{References}

1. Khan I., Ibrar A., Abbas N., Saeed A. European Journal of Medicinal Chemistry, 2013, Vol. 76, pp.193-244 doi: 10.1016/j.ejmech.2014.02.005.

2. Wang D., Gao F. Chemistry Central Journal, 2013, Vol. 7, p.95. doi:10.1186/1752-153X-7-95.

3. Bilyi A. K., Antypenko L. M., Ivchuk V. V., Kamyshnyi O. M., Polishchuk N. M., Kovalenko S. I. Chem. Plus Chem., 2015, Vol. 6, pp.980-989. doi:10.1002/ cplu.201500051.

4. Antipenko L. N., Karpenko A. V., Kovalenko S. I., Katsev A. M., Komarovska-Porokhnyavets E. Z., Novikov V. P., 2009, Vol. 342 (11), pp.651-662. doi:10.1002/ardp.200900077.

5. Špirková K., Stankovský Š., Dandárová M. Collection of Czechoslovak Chemical Communications, 1994, Vol. 59 (1), pp.222-226. doi:10.1135/cccc19940222.

6. Korshak V. V., Rusanov A. L., Iremashvili Ts G., Zhuravleva I. V., Baranov E. L. Macromolecules, 1973, Vol. 6 (4), pp.483-492. doi:10.1021/ma60034a002.

7. Gusev A. N., Shul'gin V. F., Topilova Z. M., Meshkova S. B. Russian Chemical Bulletin, Vol. 61 (1), pp.95-98. doi:10.1007/s11172-012-0014-9.

8. Karpenko A.V., Kovalenko S. I., Shishkina S. V., Shishkin O. V. Chemical Monthly, 2006, Vol. 137 (12), pp.1543-1549. doi:10.1007/s00706-006-0545-6.

9. Borioni A., Del Giudice M. R., Ferretti R., Mustazza C., Rodomonte A., Sbraccia M., Sestili I. Chemical and Pharmaceutical Bulletin; 2006, Vol. 54 (5), pp.611-622. doi:10.1248/cpb.54.611.

10. Kovalenko S. I., Antypenko L. M., Bilyi A. K., Kholodnyak S. V., Karpenko O. V., Antypenko O. M. et al. Scientia Pharmaceutica, 2013, Vol. 81 (2), pp.359391. doi:10.3797/scipharm.1211-08.

11. Kovalenko S. I, Voloshina V. O., Karpenko O. V. Zhurnal Organichnoi ta Farmatsevtichnoi Khimii - Journal of Organic and Pharmaceutical, 2010, Vol. 8 (2(80)), pp.48-56.

12. Gunther H. NMR Spectroscopy: Basic Principles, Concepts and Applications in Chemistry, 3rd Edition., Wiley, 2013, 734 p.

Надійшла до редакції 05.11.2015 p. 